\title{
Constructing Prosumer Coalitions for Energy Cost Savings Using Cooperative Game Theory
}

\author{
Liyang Han \\ Thomas Morstyn \\ Malcolm McCulloch \\ Department of Engineering Science \\ University of Oxford \\ Oxford, United Kingdom \\ \{Liyang.Han, Thomas.Morstyn, Malcolm.McCulloch\}@eng.ox.ac.uk
}

\begin{abstract}
Distributed energy storage (ES) is widely regarded as a tool to mitigate the supply-demand imbalance caused by intermittent generations and variable loads in a distribution network. Because a retail supplier generally offers a lower electricity sell price (e.g. feed-in tariff) than its buy price, ES owners are monetarily incentivized to store excess generation for later usage. However, since each ES owner typically optimizes the ES operation based on their individual usage profile, the joint load balancing effect of multiple ES systems becomes insignificant. This paper proposes an energy coalition, in which ES system owners operate collaboratively to minimize the total coalitional energy cost. Using cooperative game theory, the nucleolus and Shapley value are developed as methods to reward players. Finally, selected case studies compare the load balancing effectiveness of noncooperative and cooperative ES operations, and empirically demonstrate the nucleolus's stablizing characteristic, which incentivizes prosumers to stay within the grand coalition.
\end{abstract}

Index Terms-Cooperative game theory, energy storage, nucleolus, prosumers, Shapley value

\section{INTRODUCTION}

The advances in smart technologies are giving rise to prosumer ${ }^{1}$ energy markets in various forms, enabling prosumers to trade energy among themselves [1]. However, these market structures tend to undermine the balance of energy supply and demand, due to a lack of control and management of local energy resources.

To penalize the inflexibility of prosumer generation, retail contracts generally pay prosumers less for excess generation than they charge for energy usage. Under such electricity pricing assumption, forming local energy coalitions is proposed in [2] to quantify the economic benefit to the prosumers for having a more locally balanced energy supply and demand. Using cooperative game theory, it proves that even in the absence of energy storage (ES) systems, prosumers can gain monetary profit by forming coalitions to aggregate their production and consumption. It also proves the game is balanced [3], meaning there exists a stablizing allocation of profit that ensures no prosumers can benefit more by leaving the grand coalition to form smaller coalitions. Cooperative game theory has also been used to study ways of reducing distribution losses [4],

\footnotetext{
${ }^{1}$ proactive-consumers with distributed energy resources that actively manage their consumption and production of energy
}

and increasing the contracted energy generation price through aggregating wind power [5]. However, none of these works analyze active controls of ES operations in the power network. On the other hand, noncooperative game theory used in the distribution network is heavily focused on ES operations, mainly concerning strategies to encourage "valley filling" [6] by having EV owners operate their EV batteries to maximize their own welfare [7]-[9] on a distributed level.

Distributed ES services can be broadly grouped into four categories: energy shifting, peak shaving, power quality regulation and spinning reserve [10]. To fulfil these duties, a robust energy management strategy based on certain economic objectives is key. In this control algorithm, the variables are the ES charge/discharge energies at each timestep, and the objective is to minimize the total system cost [11]. Energy management is needed to optimally schedule the ES systems, but if they have different owners, an additional mechanism is needed to incentivize coordination.

This paper proposes the novel use of cooperative game theory to incentivize optimized ES system control for prosumer coalitions. The optimization objective is to gain the highest monetary rewards for the participants through collaborative operation of multiple ES systems, using centralized control. The case studies show that by using this collaborative control scheme, the ES systems also provide services such as peak shaving and energy shifting to increase local utilization of renewable energy.

The rest of this paper is structured as follows: Section II introduces the concept of a prosumer energy coalition and sets up the cooperative game theory framework to quantify the value of such a coalition and to reward its participants, Section III explains in detail the model implementation, including the optimization of coalitional ES operation and the computation of the payoffs. Section IV uses case studies to demonstrate the economic payback to the participants and the benefit on the power network under the proposed strategy. Section V concludes the paper.

\section{Cooperative Game Formulation}

For a prosumer who owns a distributed PV system and an ES system, the most common objective in optimizing the ES 
operation is to minimize the total energy cost. Because the retail supplier normally offers a lower sell price (e.g. feedin tariff) and charges a higher buy price for electricity, the prosumer can save money by storing the excess PV generation in the ES system and using it when the PV is generating less than they need. Considering a group of prosumers who have different load profiles and distributed energy resources, when they each optimize their own ES system to minimize their energy bill, the joint operation of all the ES systems is unlikely to yield the minimum total energy cost for the group.

This section proposes the concept of an energy coalition, a group of prosumers who collaboratively operate their ES systems to minimize the total group energy cost. A cooperative game theoretic approach is then used to analyze methods to distribute the energy cost savings among the prosumers.

Section II-A explains the concept of a coalition and defines the coalitional energy cost as a function of the ES operation. Section II-B monetizes the value of forming energy coalitions by using the coalitional energy cost. Section II-C introduces the core of a game, which consists of payoff allocation methods that incentivize all participants to stay in the grand coalition. Section II-D compares two standard payoff allocation methods.

\section{A. Prosumer Coalitional Energy Cost}

Let a group of $N$ prosumers form grand coalition $\mathcal{N}$ indexed by $i \in \mathcal{N}:=\{1,2, \ldots, N\}$. A coalition is any subset $\mathcal{S} \subseteq \mathcal{N}$. The set of all possible coalitions is defined as the power set $2^{\mathcal{N}}$ of $\mathcal{N}$. In other words, any coalition $\mathcal{S} \in 2^{\mathcal{N}}$.

For each prosumer $i$ we assume no more than one ES system. We then consider $K$ timesteps $(t=1,2, \ldots, K)$ with a time interval of $\Delta t$. We define the following:

$q_{i t}$ : Prosumer $i$ 's net energy consumption (positive) and generation (negative) without ES at timestep $t,[k W h]$

$b_{i t}$ : Prosumer $i$ 's ES system's charge (positive) and discharge (negative) energy variables at timestep $\mathrm{t},[k W h]$

$p_{t}^{b}$ : Electricity buy price at timestep $\mathrm{t}[£ / k W h]$

$p_{t}^{s}$ : Electricity sell price at timestep $\mathrm{t}[£ / k W h]$

The remuneration for distributed generation has been consistently declining in recent years, and it is assumed in this paper that $p_{b}>p_{s}$ in every timestep, as is already the case in the UK, Germany and many other countries of relatively high penetrations of distributed generation. Line losses are not accounted for here considering we are only modeling a small local power network.

For coalition $\mathcal{S} \in 2^{\mathcal{N}}$, the total energy cost is the sum of energy costs of all the prosumers within $\mathcal{S}$. Here we only consider ES systems as controllable loads. Load shifting and flexible loads are out of the scope of this paper. Therefore, we can write the the energy cost as a function of ES operation variables:

$$
F_{\mathcal{S}}(\mathbf{b})=\sum_{t=1}^{K}\left\{p_{t}^{b} \sum_{i \in \mathcal{S}}\left[q_{i t}+b_{i t}\right]^{+}+p_{t}^{s} \sum_{i \in \mathcal{S}}\left[q_{i t}+b_{i t}\right]^{-}\right\}
$$

where $[x]^{+(-)}=\max (\min )\{x, 0\}$.

We then define the coalitional energy cost for $\mathcal{S}$ as the lowest total energy cost that can be achieved by optimizing the operation of all the ES systems within $\mathcal{S}$ together:

$$
C(\mathcal{S})=\min _{\mathbf{b}} F_{\mathcal{S}}(\mathbf{b})
$$

This paper considers centralized deterministic ES control, but the specific business model is out of its scope.

\section{B. Value of Energy Coalitions}

A cooperative game analyzes situations where additional values can be achieved when players operate collaboratively. Here, we apply the standard way of analyzing a cooperative game on the $N$-prosumer energy coalition we constructed.

For each energy coalition $\mathcal{S}$ we specify a characteristic function or value function $v(\mathcal{S}): 2^{\mathcal{N}} \rightarrow \mathbb{R}$ that represents the value of $\mathcal{S}$. We define this value as the energy cost savings by forming $\mathcal{S}$, which is the difference between the sum of the minimum energy costs of each prosumer in $\mathcal{S}$ by optimizing their ES systems individually, and the minimum coalitional energy cost of $\mathcal{S}$ by optimizing the ES systems collaboratively:

$$
v(\mathcal{S})=\sum_{i \in \mathcal{S}} C(\{i\})-C(\mathcal{S})
$$

The pair $(\mathcal{N}, v)$ defines our prosumer cooperative game, and the value of the grand coalition $v(\mathcal{N})$ denotes the total amount of payoffs we can award to the prosumers.

\section{Core of the Prosumer Cooperative Game}

Having calculated the total amount of payoffs available, the next step is to determine how to allocate it to each prosumer.

Definition 1 (Imputation): We use vector $\mathbf{x} \in \mathbb{R}^{N}$ as the payoff allocation whose entry $x_{i}$ represents the payment to prosumer $i \in \mathcal{N}$. $\mathbf{x}$ is said to be an imputation if it meets both the Efficiency and Individual Rationality criteria:

1) (Efficiency) A payoff allocation $\mathrm{x}$ is said to be efficient if $\sum_{i \in \mathcal{N}} x_{i}=v(\mathcal{N})$, meaning the total amount of grand coalition's energy cost savings is allocated to the prosumers.

2) (Individual Rationality) A payoff allocation $\mathbf{x}$ is said to be individually rational if $x_{i} \geq v(\{i\}), \forall i \in \mathcal{N}$. In our game, because the value function represents the energy cost savings, $v(\{i\})=0, \forall i \in \mathcal{N}$. Therefore, this criterion guarantees that no prosumer is losing money by joining the grand coalition.

The set of all imputations for our game is defined as

$$
\mathcal{I}:=\left\{\mathbf{x} \in \mathbb{R}^{N} \mid \sum_{i \in \mathcal{N}} x_{i}=v(\mathcal{N}), x_{i} \geq v(\{i\}), \forall i \in \mathcal{N}\right\}
$$

However, an imputation does not guarantee everyone being satisfied in the grand coalition, as some players may be able to achieve higher payoffs by forming smaller energy coalitions.

Definition 2 (Excess): We measure an energy coalition's dissatisfaction with respect to the imputation $\mathrm{x}$ by its excess defined as

$$
\varepsilon(\mathbf{x}, \mathcal{S})=v(\mathcal{S})-\sum_{i \in \mathcal{S}} x_{i}
$$


When $\varepsilon(\mathbf{x}, \mathcal{S})>0$, it indicates that $\mathcal{S}$ breaking off of the grand coalition can offer higher payoffs to its members.

Definition 3 (Core): For our cooperative game $(\mathcal{N}, v)$, the core $\mathcal{C}$ is defined as the set of imputations such that no energy coalition has a value greater than the sum of its members' allocated payoffs:

$$
\mathcal{C}:=\left\{\mathbf{x} \in \mathcal{I} \mid \varepsilon(\mathbf{x}, \mathcal{S}) \leq 0, \forall \mathcal{S} \in 2^{\mathcal{N}}\right\}
$$

A payoff allocation $\mathbf{x}$ is said to be stabilizing if $\mathbf{x} \in \mathcal{C}$. However, some games have empty cores, meaning no matter how payoffs are allocated, there will always be one or more coalitions that are dissatisfied.

\section{Shapley Value vs. Nucleolus}

Two important classes of games with nonempty cores are convex games and balanced games [5].

Definition 4 (Convex Game): A game is convex if

$$
v(\mathcal{S})+v(\mathcal{T}) \leq v(\mathcal{S} \cup \mathcal{T})+v(\mathcal{S} \cap \mathcal{T}), \quad \forall \mathcal{S}, \mathcal{T} \in 2^{\mathcal{N}}
$$

which can be rewritten as

$$
\begin{aligned}
v(\mathcal{S} \cup\{i\})-v(\mathcal{S}) \leq v(\mathcal{T} \cup\{i\})-v(\mathcal{T}), & \\
& \forall i \in \mathcal{N}, \forall \mathcal{S} \subset \mathcal{T} \subset \mathcal{N} \backslash\{i\}
\end{aligned}
$$

This indicates an increasing marginal value for coalition members as the coalition grows in size. The core of a convex game is not empty, and the imputation that lies in the center of a convex game's core is the Shapley value [12]:

Definition 5 (Shapley Value): The Shapley value of a cooperative game is given by

$$
\phi_{i}(v)=\sum_{\mathcal{S} \in 2^{\mathcal{N}}, i \in \mathcal{S}} \frac{(|\mathcal{S}|-1) !(N-|\mathcal{S}|) !}{N !}[v(\mathcal{S})-v(\mathcal{S} \backslash\{i\})]
$$

The Shapley value also satisfies the following axioms:

1) (Efficiency) $\sum_{i \in \mathcal{N}} \phi_{i}(v)=v(\mathcal{N})$.

2) (Individual Rationality) $\phi_{i}(v) \geq v(\{i\}), \forall i \in \mathcal{N}$.

3) (Symmetry) If $v(\mathcal{S} \cup\{i\})=v(\mathcal{S} \cup\{j\}), \forall \mathcal{S} \in 2^{\mathcal{N}}, \mathcal{S} \cap$ $\{i, j\}=\emptyset$, then $\phi_{i}(v)=\phi_{j}(v)$.

4) (Dummy Axiom) If $v(\mathcal{S})=v(\mathcal{S} \cup\{i\}), \forall \mathcal{S} \in 2^{\mathcal{N}}, \mathcal{S} \cap$ $\{i\}=\emptyset$, then $\phi_{i}(v)=0$.

5) (Additivity) If $v$ and $u$ are characteristic functions, then $\phi_{i}(v+u)=\phi_{i}(v)+\phi_{i}(u), \forall i \in \mathcal{N}$.

Axiom 1) and 2) qualify Shapley value as an imputation. Axiom 3) says that if the marginal value of two players are the same then the Shapley values assigned to them should be equal. Axiom 4) says that if the marginal value of a player is zero then its Shapley value should also be zero. Axiom 5) says that the Shapley value of two games played at the same time should be the sum of the two games' Shapley values when played separately.

Shapley value represents the average of the incremental benefits for all possible coalitions and is considered intuitively 'fair' [13]. However, despite all the desirable properties the Shapley value holds, it is not in the core of our prosumer cooperative game, which is shown in a counterexample in Section IV. Therefore, we continue to look at balanced games, another type of game that is guaranteed to have a nonempty core [3]:

Definition 6 (Balanced Game): A map $\alpha: 2^{\mathcal{N}} \rightarrow[0,1]$ is called a balanced map if

$$
\sum_{\mathcal{S} \in 2^{\mathcal{N}}} \alpha(\mathcal{S}) 1_{i}^{\mathcal{S}}=1, \forall i \in \mathcal{N}
$$

where the indicator funtion $1_{i}^{\mathcal{S}}=\left\{\begin{array}{ll}1, & \text { if } i \in \mathcal{S} \\ 0, & \text { otherwise }\end{array}\right.$.

A cooperative game is said to be balanced if for any balanced map $\alpha$,

$$
\sum_{\mathcal{S} \in 2^{\mathcal{N}}} \alpha(\mathcal{S}) v(\mathcal{S}) \leq v(\mathcal{N})
$$

Definition 7 (Nucleolus): We denote $\epsilon(\mathbf{x}) \in \mathbb{R}^{2^{N}-2}$ as the vector whose entries are the excesses in respect to imputation $\mathbf{x}$ for all coalitions (excluding the grand coalition) arranged in a nonincreasing order. The nucleolus $\nu$ is an imputation such that

$$
\epsilon_{j}(\nu) \leq \epsilon_{j}(\mathbf{x}), \forall \mathbf{x} \in \mathcal{I}, \forall j \in 2^{N}-2
$$

This means that the nucleolus of a cooperative game is the lexicographically minimal imputation that minimizes the dissatisfaction of the players [5]. The nucleolus always exists, and for a game with a nonempty core (balanced), the nucleolus always belongs to the core [14], hence is a stabilizing imputation. in Section IV, we demonstrate through a considerable number of simulations that the largest excess of the nucleolus is always nonpositive, indicating our prosumer cooperative game is likely a balanced game.

\section{CoOperative Game Model IMPlementation}

To implement this prosumer cooperative model, we

1) Collect model inputs, including the number of prosumers, each prosumer's load and generation profiles, ES system information specified in Section III-A, and the electricity buy and sell prices.

2) Generate a list of all possible energy coalitions $\left(2^{\mathcal{N}}\right)$, and optimize the coalitional ES operation to minimize each coalitional energy cost, calculate the value of each energy coalition using (3). This step is further explained in Section III-A.

3) Compute the desired imputation (e.g. Shapley value, nucleolus) using the coalition values calculated in the previous step, and examine its excesses to determine whether the imputation is stabilizing. This step is further explained in Section III-B.

\section{A. Energy Storage Optimization}

In order to compute the coalitional energy cost for $\mathcal{S} \in 2^{\mathcal{N}}$ in (2), we need to constrain the ES operation variables properly. Therefore, we consider each prosumer $i$ 's ES system has an energy capacity of $e_{i} \in \mathbb{R}_{\geq 0}$, in $[k W h]$, a charge limit and a discharge limit over the time span of $\Delta t$ of $\bar{b}_{i} \in \mathbb{R}_{\geq 0}$ and $\underline{b}_{i} \in \mathbb{R}_{\geq 0}$, in $[k W h]$, a charge efficiency and a discharge efficiency of $\eta_{i}^{\text {in }}$ and $\eta_{i}^{\text {out }} \in(0,1)$, and an 
initial state of charge of $S o C_{i}^{0} \in[0,1]$. Using parameters and variables defined in Section II-A, for $\mathcal{S} \in 2^{\mathcal{N}}$, the ES operation variables $\mathbf{b}$ are subject to:

1) Power constraint: the charge and discharge energy for each timestep is constrained by the charge and discharge limit:

$$
\underline{b}_{i} \leq b_{i t} \leq \bar{b}_{i}, \quad \forall i \in \mathcal{S}, \forall t \in[1, K]
$$

We set $\underline{b}_{i}=\bar{b}_{i}=0$ for prosumers that do not own an ES system.

2) Energy constraint: the energy stored is limited between zero and each ES system's energy capacity for every timestep:

$$
\begin{aligned}
& 0 \leq e_{i} S o C_{i}^{0}+\sum_{t=1}^{k}\left(\left[b_{i t}\right]^{+} \eta_{i}^{i n}+\left[b_{i t}\right]^{-} / \eta_{i}^{\text {out }}\right) \leq e_{i} \\
& \forall i \in \mathcal{S}, \forall k \in[1, K]
\end{aligned}
$$

3) Cycle constraint: the state of charge at $t=K$ is required to be restored to $S o C_{i}^{0}$ for every ES system:

$$
\sum_{t=1}^{K}\left(\left[b_{i t}\right]^{+} \eta_{i}^{\text {in }}+\left[b_{i t}\right]^{-} / \eta_{i}^{\text {out }}\right)=0, \quad \forall i \in \mathcal{S}
$$

In this paper, due to the short time frame ( 24 hours in the case studies) being analyzed, we assume no battery selfdischarge and no battery degradation. To obtain a linear battery optimization problem, a piecewise linear battery model with a constant charging efficiency and a constant discharging efficiency is commonly used [15]. In this paper, we define the discharge and charge energies as $\mathbf{b}^{-}$and $\mathbf{b}^{+}$respectively, to avoid using the binary variable [16] while still maintaining the model's linearity.

Similarly, there is a piecewise function in the objective function due to the different buy and sell prices for electricity, so we rewrite the coalitional net load into a positive part $\mathbf{L}^{+}$ and a non-positive part $\mathbf{L}^{-}$, adapting a method from [17].

Rewriting (1) and (2) in a linear format:

$$
\begin{aligned}
\min _{\substack{\mathbf{b}^{+}, \mathbf{b}^{-}, \mathbf{L}^{+}, \mathbf{L}^{-}}} & \sum_{t=1}^{K}\left\{p_{t}^{b} \sum_{i \in \mathcal{S}} L_{i t}^{+}+p_{t}^{s} \sum_{i \in \mathcal{S}} L_{i t}^{-}\right\} \\
\text {s.t. } & L_{i t}^{-} \leq 0 \leq L_{i t}^{+} \\
& b_{i t}^{+}+b_{i t}^{-}+q_{i t} \leq L_{i t}^{+} \\
& b_{i t}^{+}+b_{i t}^{-}+q_{i t}=L_{i t}^{+}+L_{i t}^{-} \\
& 0 \leq b_{i t}^{+} \leq \bar{b}_{i} \\
& \underline{b}_{i} \leq b_{i t}^{-} \leq 0 \\
& 0 \leq e_{i} S o C_{i}^{0}+\sum_{t=1}^{k}\left(b_{i t}^{+} \eta_{i}^{i n}+b_{i t}^{-} / \eta_{i}^{o u t}\right) \leq e_{i} \\
& \sum_{t=1}^{K}\left(b_{i t}^{+} \eta_{i}^{i n}+b_{i t}^{-} / \eta_{i}^{o u t}\right)=0 \\
& \forall i \in \mathcal{S}, \forall t \in[1, K], \forall k \in[1, K]
\end{aligned}
$$

Constraints (5), (6) and the minimization objective function effectively set $L_{i t}^{+}=\max \left\{0, b_{i t}^{+}+b_{i t}^{-}+q_{i t}\right\}$, which, along with (7), sets $L_{i t}^{-}=\min \left\{0, b_{i t}^{+}+b_{i t}^{-}+q_{i t}\right\}$. The new power constraint is denoted by (8) and (9), the new energy constraint (10), and the new cycle constraint (11). Due to the charge, discharge efficiencies $(<1)$ and the cost minimization, the optimization process will make sure not to have $b_{i t}^{+}$and $b_{i t}^{-}$ taking on non-zero values at the same timestep.

\section{B. Computation of Shapley Value and Nucleolus}

After a complete list of coalition values is generated using (3), the Shapley value can be easily calculated using (4).

To solve for the nucleolus of our prosumer cooperative, we follow the iterative process below [18]:

1) Solve the following linear optimization:

$$
\begin{aligned}
L P_{1}: \varepsilon_{1}= & \min _{\mathbf{x}, \varepsilon} \varepsilon \\
\text { s.t. } & \sum_{\forall i \in \mathcal{N}} x_{i}=v(\mathcal{N}) \\
& v(\mathcal{S})-\sum_{\forall i \in \mathcal{S}} x_{i} \leq \varepsilon, \forall \mathcal{S} \notin\{\emptyset, \mathcal{N}\}
\end{aligned}
$$

where Constraint (13) ensures the efficiency criterion of the imputation, Constraint (14) sets $\varepsilon$ as the largest excess for all coalitions, and the objective function (12) minimizes the largest excess by searching through possible imputations. We obtain optimal solution $\varepsilon_{1}$, and $\mathfrak{S}_{1}$, a set of coalitions that are common to all optimal solutions at $\varepsilon=\varepsilon_{1}$, or coalitions that correspond to constraints defined by (14) that are binding. $\varepsilon_{1}$ is the largest excess of the nucleolus although the nucleolus has not been completely computed by this time. Therefore, if $\varepsilon_{1} \leq 0$, the nucleolus is in the core.

2) Iteratively solve the following linear optimization until a unique optimal solution $\mathrm{x}^{*}$ is obtained. $\mathrm{x}^{*}$ then is the nucleolus of our prosumer cooperative game.

$$
\begin{array}{ll}
L P_{j}: \varepsilon_{j}= & \min _{\mathbf{x}, \varepsilon} \varepsilon \\
\text { s.t. } & \sum_{\forall i \in \mathcal{N}} x_{i}=v(\mathcal{N}) \\
& \sum_{\forall i \in \mathcal{S}} x_{i}=v(\mathcal{S})+\varepsilon_{l}, \quad \forall \mathcal{S} \in \mathfrak{S}_{l}, \\
& \forall l \in[1, j-1] \\
& v(\mathcal{S})-\sum_{\forall i \in \mathcal{S}} x_{i} \leq \varepsilon, \quad \forall \mathcal{S} \notin\left\{\emptyset, \mathfrak{S}_{l}, \mathcal{N}\right\}, \\
& \forall l \in[1, j-1]
\end{array}
$$

where $j \geq 2$, numbering each linear program sovled during the iteration process. Constraint (15) ensures the efficiency criterion of the imputation, Constraint (16) fixes the excesses of the coalitions that are binding in all previous iterations, Constraint (17) sets $\varepsilon$ as the largest excess for all remaining coalitions in $L P_{j} . \mathfrak{S}_{l}:=\left\{\mathcal{S} \mid v(\mathcal{S})-\sum_{\forall i \in \mathcal{S}} x_{i}=\right.$ $\varepsilon_{l}, \forall$ optimal decision variable $\mathbf{x}$ in $\left.L P_{l}\right\}$, which represents the set of coalitions whose excesses are binding based on (17) in $L P_{l}$. 


\section{CAse Studies}

In this section, we test our prosumer cooperative game using European Low Voltage Test Feeder load data published by IEEE Power and Energy Society [19], and demonstrate that the energy coalition does help reduce reverse power flow in the distributed network and smoothen the load profile locally. We then compare the Shapley value and the nucleolus to show that both imputations are able to reward all players, but only the nucleolus is a stabilizing imputation in all tested examples.

In the case studies, we select a time frame of 24 hours starting from the midnight of a sunny autumn day in October. The battery model is one with an energy capacity of $7 \mathrm{kWh}$, a maximum charge power of $3.5 \mathrm{~kW}$, a maximum discharge power of $3.2 \mathrm{~kW}$, both charge and discharge efficiencies of $95 \%$, and an initial state of charge of 50\%. The PV generation data are simulated in PVWatts using a $4 \mathrm{~kW}$, fixed 20 degree tilt residential system, and the London Gatwick solar data [20]. The energy buy price follows a UK Economy 7 residential rate structure: $£ 0.072 / \mathrm{kWh}$ for midnight $-7 \mathrm{am}$, and $£ 0.1681 / \mathrm{kWh}$ for 7am-midnight [21], and the energy sell price is the UK feed-in tariff [22] fixed at $£ 0.0485 / \mathrm{kWh}$.

\section{A. Effective Load Balancing}

For the first case study, we select 12 prosumers, $6 \mathrm{PV}$ systems and 6 batteries, which are randomly assigned to the prosumers. Fig. 1 compares three different coalitional load profiles: 1) the coalitional load profile without operating any ES system, 2) the coalitional load profile with ES systems operating noncooperatively to minimize each of their owner's energy cost, and 3) the coalitional load profile with ES systems operating cooperatively to minimize the coalitional energy cost. It can be easily seen in the graph that ES operating noncooperatively slightly reduces the peak power and reverse power flow, while having ES operating cooperatively not only reduces the peak demand, but almost completely eliminates reverse power flow.

Fig. 2 further shows how ES operation corresponds to the raw load of the coalition both under a noncooperative scheme and a cooperative one. Both ES operations tend to reverse the load by discharging (negative) when there is an energy demand and charging (positive) when there is a generation excess, but the cooperative ES operation demonstrates a clearer pattern of following, nearly perfectly mirroring, the load profile.

\section{B. Nucleolus vs. Shapley Value}

For the second case study, we run the model with different numbers $(N=4,6,8,10,12,14)$ of players. In each run, we assume $50 \%$ of the prosumers own a PV, and 50\% ES system, randomly assigned. We run each model 30 times and graph the average largest excess against the number of players in Fig. 3. It is shown that regardless of the number of prosumers in the grand coalition, the largest excess with respect to the Shapley value can always exceed zero, proving the statement in Section II-D that the Shapley value is not in the core of our prosumer cooperative game. On the other hand, the largest excess with

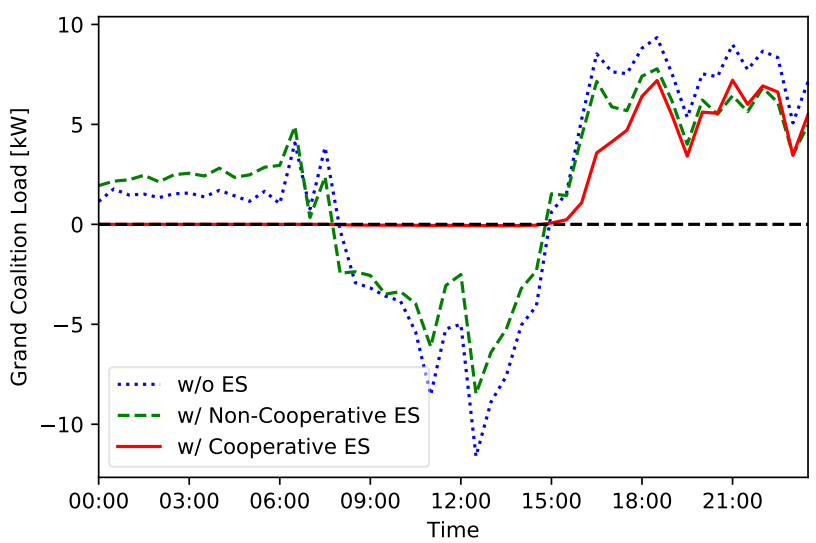

Figure 1: 12-prosumer energy coalition 24-hour load profile 1) without ES, 2) with noncooperative ES, and 3) with cooperative ES.

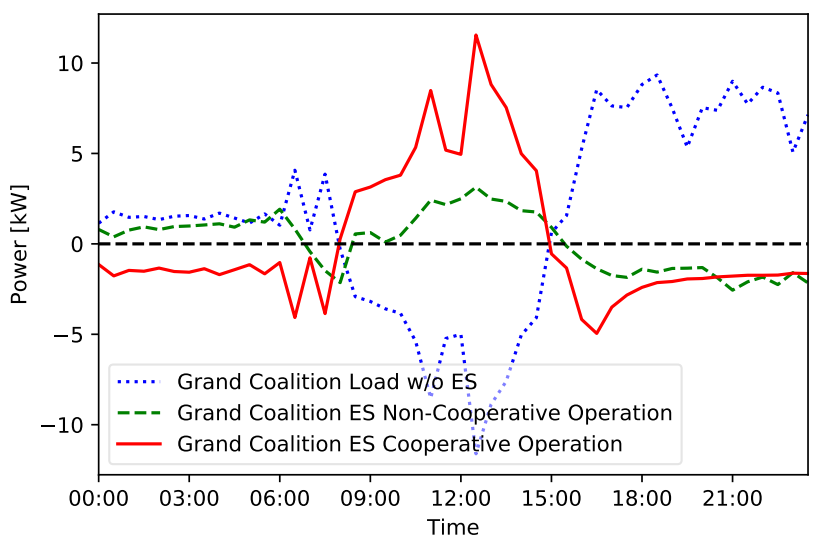

Figure 2: 12-prosumer energy coalition 24-hour total ES charging and discharging profile vs. coalition load profile.

respect to the nucleolus is non-positive in all 180 simulations, which empirically shows that the nucleolus is stabilizing.

Taking a 14-prosumer run as an example, Fig. 4 maps the excesses of all the coalitions on a nucleolus vs. Shapley coordinate. The data points generally follow the diagonal line representing "Nucleolus Excess = Shapley Excess", but some Shapley excesses exceed zero, while no nucleolus excesses do.

Fig. 5 shows the comparison of the nucleolus and Shapley allocation for each prosumer that participates in the cooperative game. Note that these payoff allocations represent the additional amount of money each prosumer receives on top of the savings they may achieve from operating their ES system, if any, to minimize their own energy cost. The figure shows that although the payoffs for each player vary slightly with the allocation method (nucleolus or Shapley), their relative values among all the players follow a similar pattern. This is because both imputations reward players on a merit basis whoever contributes more to reducing the coalitional energy cost tends to get more in return. We also notice that whether a player receives more under the nucleolus or the Shapley is dependent on its resource mix. For example, players without any PV or ES tend to be rewarded more by the nucleolus, while players with both PV and ES tend to be rewarded more 


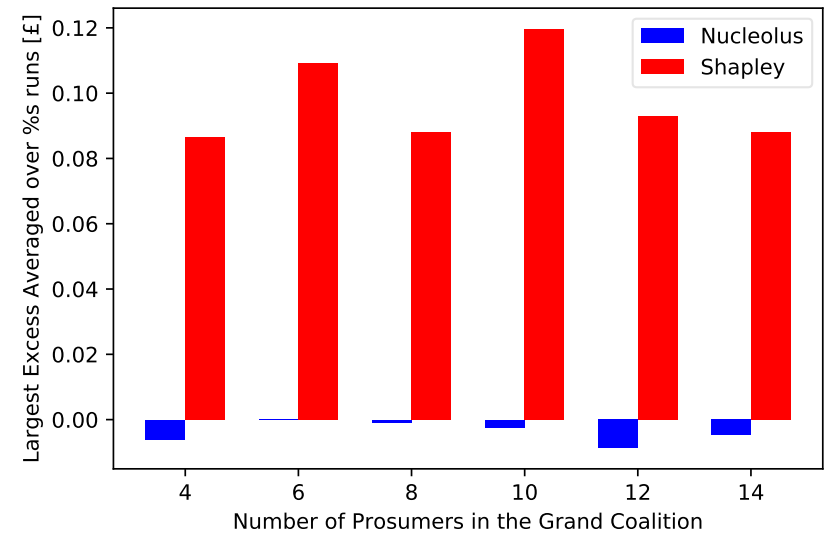

Figure 3: Largest excess under an imputation - Nucleolus vs. Shapley

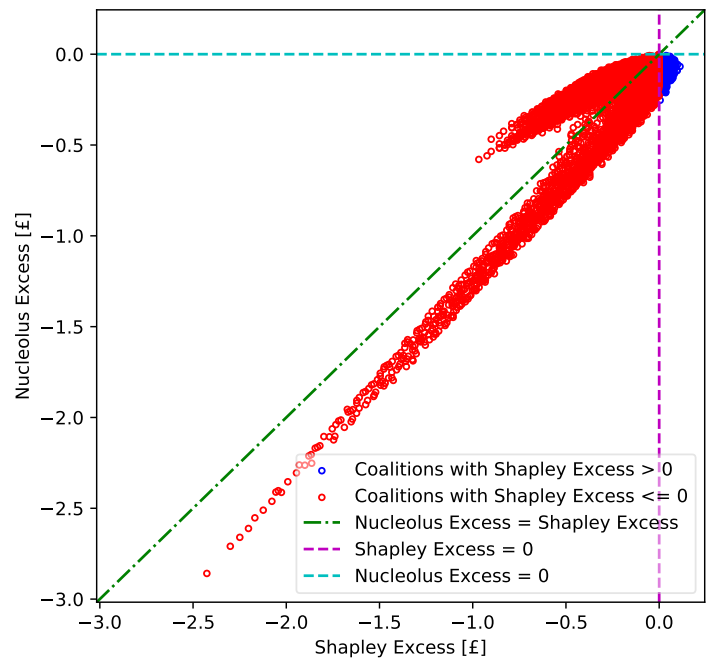

Figure 4: Nucleolus Excess vs. Shapley Excess in a 14-prosumer cooperative game

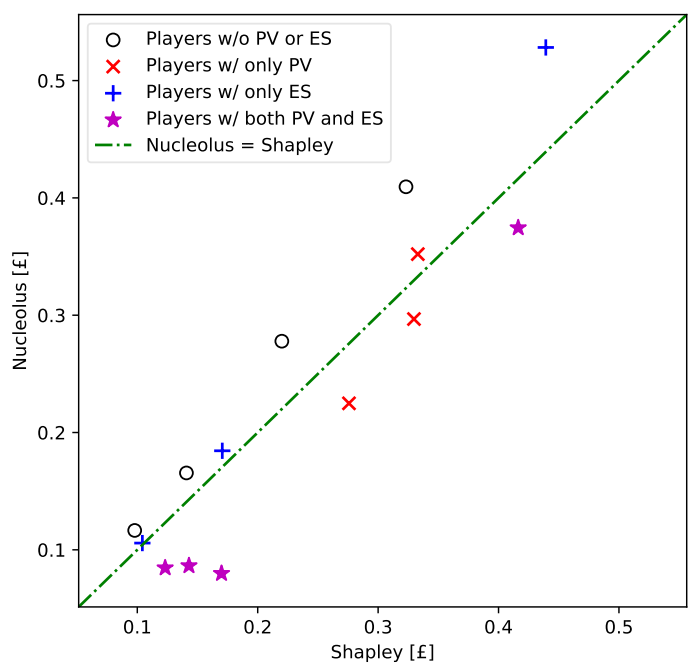

Figure 5: Nucleolus vs. Shapley value in a 14-prosumer cooperative game by the Shapley. This is likely due to the high penetration of $\mathrm{PV}$, where pure load consumption is deemed more valuable.

\section{CONCLUSION}

Using a cooperative game theoretic approach, we propose a distributed prosumer energy grand coalition, which cooperatively optimizes all the ES system operations within the coalition to minimize the total coalitional energy cost. As a result, this operational strategy reduces the reverse power flow in the distributed network and flatten the energy profile on a local level. Adapting existing ES control algorithms, we have formulated a linear problem to optimize the operation of multiple ES systems. The values of all possible coalitions within the grand coalition are defined by the coalitional energy cost savings, which can be quantified using the proposed ES optimization algorithm. Through case studies, we demonstrate empirically that the nucleolus, a way to allocate payoffs, is superior to the Shapley value in financially incentivizing prosumers to stay in the grand coalition.

The immediate extension of this work is the mathematical proof of the balancedness of our prosumer cooperative game, which will set the stabilizing allocation given by the nucleolus as a baseline to assess other allocation methods. In order to implement the said cooperative game for larger size prosumer coalitions, the development of sampling algorithms and more efficient nucleolus calculation methods to reduce the computational burden is needed. Subsequently, how the size of prosumer coalitions influences the regulatory frameworks and energy market prices is a promising area for future work.

\section{REFERENCES}

[1] Y. Parag and B. K. Sovacool, "Electricity market design for the prosumer era," Nature Energy, no. March, p. 16032, 2016.

[2] W. Lee, L. Xiang, R. Schober, and V. W. S. Wong, "Direct electricity trading in smart grid: A coalitional game analysis," IEEE Journal on Selected Areas in Communications, vol. 32, no. 7, pp. 1398-1411, 2014.

[3] L. Shapley, On Balanced Sets and Cores, ser. Memorandum (Rand Corporation). Rand Corporation, 1965.

[4] W. Saad, Z. Han, and H. V. Poor, "Coalitional game theory for cooperative micro-grid distribution networks," IEEE International Conference on Communications, pp. 6-10, 2011.

[5] E. Baeyens, E. Y. Bitar, P. P. Khargonekar, and K. Poolla, "Coalitional aggregation of wind power," IEEE Transactions on Power Systems, vol. 28, no. 4, pp. 3774-3784, 2013.

[6] D. S. Callaway and I. A. Hiskens, "Achieving Controllability of Electric Loads," Proceedings of the IEEE, vol. 99, no. 1, 2010.

[7] L. Deori, K. Margellos, and M. Prandini, "On the connection between Nash equilibria and social optima in electric vehicle charging control games," 20th World Congress of the International Federation of Automatic Control, 2017.

[8] Z. J. Ma, D. S. Callaway, and I. A. Hiskens, "Decentralized Charging Control of Large Populations of Plug-in Electric Vehicles," IEEE Transactions on Control Systems Technology, vol. 21, no. 1, pp. 67-78, 2013.

[9] B. G. Kim, S. Ren, M. Van Der Schaar, and J. W. Lee, "Bidirectional energy trading for residential load scheduling and electric vehicles," Proceedings - IEEE INFOCOM, vol. 31, no. 7, pp. 595-599, 2013

[10] T. Morstyn, B. Hredzak, and V. G. Agelidis, "Control Strategies for Microgrids with Distributed Energy Storage Systems: An Overview," IEEE Transactions on Smart Grid, vol. 3053, no. c, pp. 1-1, 2016.

[11] M. Nick, R. Cherkaoui, and M. Paolone, "Optimal allocation of dispersed energy storage systems in active distribution networks for energy balance and grid support," IEEE Transactions on Power Systems, vol. 29, no. 5, pp. 2300-2310, 2014. 
[12] L. S. Shapley, "Cores of convex games," International Journal of Game Theory, vol. 1, no. 1, pp. 11-26, 1971 .

[13] E. Faria, L. A. Barroso, R. Kelman, S. Granville, and M. V. Pereira, "Allocation of firm-energy rights among hydro plants: An AumannShapley approach," IEEE Transactions on Power Systems, vol. 24, no. 2, pp. 541-551, 2009.

[14] T. S. H. Driessen, Cooperative Games, Solutions and Applications, ser. Theory and decision library: Series C, Game theory, mathematical programming, and operations research. Springer, 1988.

[15] M. Gulin, J. Matusko, and M. Vasak, "Stochastic model predictive control for optimal economic operation of a residential DC microgrid," IEEE International Conference on Industrial Technology (ICIT), pp. 505-510, 2015.

[16] E. G. L. Parisio A.; Rikos, "A Model Predictive Control Approach to Microgrid Operation Optimization," Control Systems Technology, IEEE Transactions, vol. 1, no. 4, pp. 1813-1827, 2014.

[17] E. Perez, H. Beltran, N. Aparicio, and P. Rodriguez, "Predictive power control for PV plants with energy storage," IEEE Transactions on Sustainable Energy, vol. 4, no. 2, pp. 482-490, 2013.

[18] J. K. Sankaran, "On finding the nucleolus of an n-person cooperative game," International Journal of Game Theory, vol. 19, no. 4, pp. 329$338,1991$.

[19] IEEE-PES. (2017) Distribution test feeders. [Online]. Available: http://ewh.ieee.org/soc/pes/dsacom/testfeeders/index.html [Accessed: 2017-08-03].

[20] Pvwatts.nrel.gov. Pvwatts calculator. [Online]. Available: http://pvwatts.nrel.gov/pvwatts.php [Accessed: 2017-08-03].

[21] GOV.UK. (2017, June) Annual domestic energy bills. [Online]. Available: https://www.gov.uk/government/statistical-data-sets/annualdomestic-energy-price-statistics [Accessed: 2017-08-03].

[22] GOV.UK. (2016, Feb.) Feed-in tariffs: get money for generating your own electricity. [Online]. Available: https://www.gov.uk/feed-intariffs/overview [Accessed: 2017-08-03]. 